\title{
Temperature evaluation in steel fire protected elements with intumescent coating
}

\author{
L. M. R. Mesquita ${ }^{1}$, P. A. G. Piloto ${ }^{1}$ \& M. A. P. Vaz ${ }^{2}$ \\ ${ }^{1}$ Polytechnic Institute of Bragança, Portugal \\ ${ }^{2}$ University of Porto, FEUP, Portugal
}

\begin{abstract}
Heat transfer analysis plays an important role in the temperature prediction of insulated steel members exposed to fire conditions. Based on the results of a previous experimental work, made in intumescent-coated protected elements, the intumescent effective thermal conductivity temperature variation was estimated. An analytical approach, based on the one-dimensional heat transfer parabolic partial differential equation through the insulation, considering a non-homogeneous (time-varying) boundary condition at fire interface and a lumped capacitance at the insulation-steel interface, is presented. Solutions are established with temperature independent thermal properties and then extended to temperature dependent thermal properties. These solutions are compared with numerical results performed by finite element method using Matlab for different values of insulations thicknesses and steel section factors.

Keywords: fire resistance, fire protection, intumescent coatings, heat transfer, Duhamel's theorem, Green's function.
\end{abstract}

\section{Introduction}

Fire protection costs may represent an important part of global construction costs, being more significant in the case of steel construction, requiring frequently the application of an insulation material (Longton et al [1]). The use of passive protection materials is one of the measures normally adopted to prescribe structural fire resistance. Intumescent coating represents around one third of steel fire protection and is growing mainly due to their use in off-site applications.

The fire resistance of an insulated steel member is determined by assessing the loadbearing capacity of the component during fire exposure. Heat transfer 
analysis of the insulated steel member is of great importance for determining, accurately, critical temperature of the protected element which depends essentially on the protection material properties and on the bulk fire temperature.

The specification of the minimum protection thickness is recommended by manufactures. These values are based on experimental fire tests, using typical structural elements (beams and columns) being the reports kept confidential. The results are presented in form of tables or graphs, for different critical temperatures, protection thicknesses and different fire resistance periods. In a previous work, a set of experimental fire resistance tests was conducted on steel beams protected with intumescent coatings and the effective thermal conductivity was determined as function of the mean insulation temperature evolution (Mesquita et al [2]).

The aim of this paper is to present an analytical development that considers one-dimensional heat transfer equation using Duhamel's theorem and Green's functions methods to determine steel temperature of protected structural elements. The experimental results are compared with the analytical results and with numerical results based on the finite element method from the PDEToolbox of Matlab, considering the effective thermal conductivity temperature dependent. The study is extended to other protection thicknesses and steel section factors.

\section{Experimental fire resistance results}

The experimental results of full-scale fire tests, conducted on steel beams protected with solvent-based intumescent coating were previously published. Specimens have been coated and heated with a heating rate of $800\left[{ }^{\circ} \mathrm{C} / \mathrm{h}\right]$ and temperatures recorded on steel IPE100 profiles. Higher thickness of protection results in higher fire resistance time, increasing critical temperature.

The tests herein considered are tests 5,6 and 7, with dry film thickness mean value of 1329,1441 and $1521[\mu \mathrm{m}]$, respectively. The experimental results are presented in the figures $3-5$.

According to the Eurocode 3 formulae CEN [3], steel temperature prediction is calculated using a numerical time stepping scheme, given by:

$$
\Delta T_{S}=\frac{k_{i} P / A\left(T_{g}-T_{S}\right)}{c_{s} \rho_{s} d_{i}(1+\mu / 3)} \Delta t-\left(e^{\phi / 10}-1\right) \Delta T_{g}
$$

where $\Delta t \leq 30[\mathrm{~s}]$ for insulated steel elements. $\Delta T_{S}$ and $\Delta T_{g}$ represent the temperature increments of the insulated steel and fire gas during the time $\Delta t . P / A$ is the steel section factor, while $d_{i}$ and $k_{i}$ accounts for the thickness and conductivity of the protection material and $\mu$ is the ratio of the heat capacitance between insulation and steel. $\rho_{S}$ and $c_{S}$ represent steel specific mass and specific heat.

The inverse of eqn (1) may explicit thermal conductivity, according to temperature measured results on beam and on the heating source. This lead to the following expression: 


$$
k_{i}=\left[\frac{c_{S} \rho_{s}(1+\phi / 3)}{\left(T_{g}-T_{S}\right) \Delta t} \frac{d_{i}}{P / A}\right]\left[\Delta T_{S}+\left(e^{\phi / 10}-1\right) \Delta T_{g}\right]
$$

The effective thermal conductivity temperature variation may be approximated considering a protection temperature calculated by the average value of the heating source and steel temperature $T_{i}(i)=\left[T_{g}(i)+T_{S}(i-1)\right] / 2$ (Wickström [4]). Figure 1 represents the effective thermal conductivity determined using the experimental results and eqn (2).

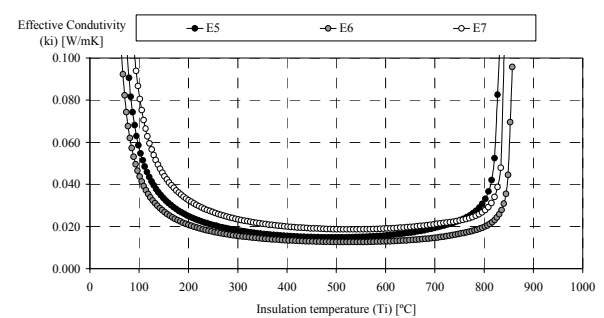

Figure 1: Effective thermal conductivity.

The shape and the resulting values are in accordance with other reported results for different intumescent material (Tan et al [5]).

\section{Analytical steel temperature development}

Heat transfer analysis of fire protected steel elements normally requires the solution of a two-dimensional transient diffusion equation. Since heating conditions assumes steel members fully embedded in fire, the 2D problem can be approximated by the one-dimensional differential conduction equation, assuming a steel lumped capacitance, see fig. 2, (Wang et al [6]). The steel thickness $d_{S}$ may be determined, inverting the steel section factor $P / A$.

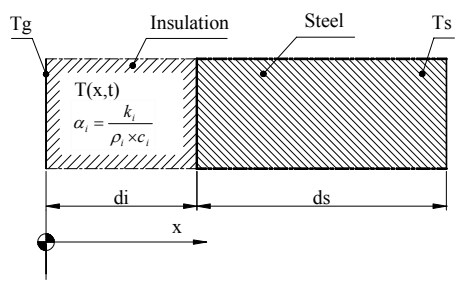

Figure 2: One-dimensional heat transfer model.

Considering constant thermal properties, temperature independent, the temperature field through the homogeneous insulation layer is given by the differential eqn (3). 


$$
\alpha_{i} \frac{\partial^{2} T(x, t)}{\partial x^{2}}=\frac{\partial T(x, t)}{\partial t}
$$

where $\alpha_{i}=k_{i} / \rho_{i} c_{i}$ represents the insulation thermal diffusivity, $\rho_{i}$ and $c_{i}$ represents the specific mass and specific heat, respectively.

The non-homogeneous boundary conditions for this model are given by:

$$
\begin{aligned}
T(0, t) & =\operatorname{Tg}(t), \quad x=0 \\
-k_{i} \frac{\partial T\left(d_{i}, t\right)}{\partial x} & =Q_{S} \frac{\partial T\left(d_{i}, t\right)}{\partial t}, \quad x=d_{i}
\end{aligned}
$$

where $Q_{S}$ represents the steel heat capacitance, $Q_{S}=\rho_{S} c_{S} d_{S}$. The initial condition for the heat transfer analysis is assumed to be:

$$
T(x, 0)=T_{0}, \quad t=0
$$

The existence of the non-homogenous (time-varying) boundary condition, eqn (4) at fire interface turns this problem impossible to be solved by classical methods. The non-classical solution uses Duhamel's theorem and Green's function, herein presented.

\subsection{Temperature development using Duhamel's theorem}

Duhamel's theorem allows expressing the solution of this problem in terms of an integral auxiliary problem solution corresponding to homogeneous boundary conditions. Applying Duhamel's theorem, if $v=F(r, t)$ represents the temperature at position $r$, for the instant $t$, in a solid with zero temperature conditions, while its surface is kept at unit temperature, the solution for the problem, when the surface temperature is $\phi(t)$, is given by eqn (7), Carslaw and Jaeger [7].

$$
v=\int_{0}^{t} \phi(\tau) \frac{\partial}{\partial t} F(r, t-\tau) d \tau
$$

Consider a new auxiliary problem defined by eqns (8-11), obtained with a variable change, $\theta(x, t)=T(x, t)-T_{0}$.

$$
\begin{gathered}
\alpha_{i} \frac{\partial^{2} \theta(x, t)}{\partial x^{2}}=\frac{\partial \theta(x, t)}{\partial t} \\
\theta(0, t)=\theta_{0}, \quad x=0 \\
-k_{i} \frac{\partial \theta\left(d_{i}, t\right)}{\partial x}=Q_{S} \frac{\partial \theta\left(d_{i}, t\right)}{\partial t}, \quad x=d_{i} \\
\theta(x, 0)=0, \quad t=0
\end{gathered}
$$

The solution to this auxiliary problem is given by the eqn (12) (Carslaw and Jaeger [7]).

$$
\frac{\theta(x, t)}{\theta_{0}}=1-\sum_{n=1}^{\infty} \frac{2\left(\beta_{n}^{2}+\mu^{2}\right)}{\beta_{n}\left(\beta_{n}^{2}+\mu^{2}+\mu\right)} e^{-\frac{\alpha_{i} \beta_{n}^{2}}{d_{i}^{2}} t} \sin \left(\frac{\beta_{n} x}{d_{i}}\right)
$$


in which $\beta_{n}$ represents the roots of the transcendental equation $\beta_{n} \operatorname{tg} \beta_{n}=\mu$. Considering the fire gas temperature given by the standard fire ISO curve, the solution of the previous integral is not straightforward, because of the logarithmic and exponential function product, appearing in the exponential integral. The common way to suppress this difficulty is to approximate the standard fire ISO curve by a sum of exponential functions, also used in the SP approach, Wang et al [6], and defined by eqn (13), regarding an initial zero temperature.

$$
\operatorname{Tg}(t)=\sum_{j=0}^{3} \theta_{j} e^{-\phi_{j} t}
$$

The coefficients $\theta_{j}$ and $\phi_{j}$ are constants reproduced in table 1 .

Table 1: Constants in exponential expression.

\begin{tabular}{lllll}
\hline$j$ & 0 & 1 & 2 & 3 \\
\hline$\theta\left[{ }^{\mathrm{o}} C\right]$ & 1325 & -430 & -270 & -625 \\
\hline$\phi\left[h^{-1}\right]$ & 0 & 0.2 & 1.7 & 19 \\
\hline
\end{tabular}

The solution of the integral eqn (7) considering eqn (13) allows the determination of steel time varying temperature, considering $x=d_{i}$.

$$
T\left(d_{i}, t\right)=T_{s}(t)=T_{0}+\sum_{n=1}^{\infty} \sum_{j=0}^{3}\left[\frac{\theta_{j}\left(e^{\left(-\phi_{j}\right) t}-e^{-\frac{\alpha_{i}}{d_{i}{ }^{2}}{ }^{2} t}\right)}{1-\phi_{j} d_{i}{ }^{2} /\left(\alpha_{i} \beta_{n}{ }^{2}\right)} K_{n} \sin \left(\beta_{n}\right)\right]
$$

where $K_{n}=2\left(\beta_{n}^{2}+\mu^{2}\right) /\left[\beta_{n}\left(\beta_{n}^{2}+\mu^{2}+\mu\right)\right]$.

\subsection{Temperature development using Green's function}

Consider, for general proposes, the heat transfer problem defined by eqn (15) in region $R$, subjected to the generic boundary condition eqn (16) at surface $S_{i}$ and initial condition eqn (17) (Beck [9]).

$$
\begin{gathered}
\nabla^{2} T(r, t)+\frac{1}{k} g(r, t)-m^{2} T=\frac{1}{\alpha} \frac{\partial T(r, t)}{\partial t} \\
k_{i} \frac{\partial T}{\partial n_{i}}+h_{i} T=f_{i}\left(r_{i}, t\right)-(\rho c d)_{i} \frac{\partial T}{\partial t} \\
T(r, 0)=F(r)
\end{gathered}
$$


The term $m^{2} T$ could represent the side heat losses being $m=f(r)$. The general boundary condition may represent five different boundary conditions by setting $k_{i}=0$ or $k, h_{i}=0$ or $h$ and $d=0$ or $d \neq 0$.

The Green's function (GF) method consists to define the GF to determine the temperature solution. The GF, $G\left(r, t \mid r^{\prime}, \tau\right)$, represents the temperature at the location $r$ and time $t$, resulting from an instantaneous point source of heat releasing a unit of thermal energy at location $r^{\prime}$ and time $\tau$.

The GF associated with the problem defined by eqns (15-17) obeys the following equations, where $\nabla_{0}^{2}$ relates to the $r^{\prime}$ coordinates.

$$
\begin{gathered}
\nabla_{0}^{2} G+\frac{1}{\alpha} \delta\left(r^{\prime}-r\right) \delta(\tau-t)-m^{2} G=\frac{1}{\alpha} \frac{\partial G}{\partial \tau} \\
k_{i} \frac{\partial G}{\partial n_{i}}+h_{i} G=(\rho c d)_{i} \frac{\partial G}{\partial \tau}=Q_{S i} \frac{\partial G}{\partial \tau} \\
G\left(r, t \mid r^{\prime}, \tau\right)_{\tau=0}=0
\end{gathered}
$$

$\delta$ represents Dirac's delta function. This auxiliary problem has homogeneous boundary conditions and zero initial temperature. Since the GF may only be found for linear differential equations, the thermal properties are kept constant. Rewriting eqn (15) in terms of $r^{\prime}$ and $\tau$, results.

$$
\nabla_{0}^{2} T\left(r^{\prime}, \tau\right)+\frac{1}{k} g\left(r^{\prime}, \tau\right)-m^{2} T\left(r^{\prime}, \tau\right)=\frac{1}{\alpha} \frac{\partial T\left(r^{\prime}, \tau\right)}{\partial \tau}
$$

The product of the eqn (21) by $G$ and eqn (18) by $T$, subtracting the last from the previous, the following equation is obtained.

$$
\begin{aligned}
& \left(G \nabla_{0}^{2} T-T \nabla_{0}^{2} G\right)+\frac{1}{k} g\left(r^{\prime}, \tau\right) G-\frac{1}{\alpha} \delta\left(r^{\prime}-r\right) \delta(\tau-t) T \\
& -m^{2}(G T-T G)=\frac{1}{\alpha} \frac{\partial(T G)}{\partial \tau}
\end{aligned}
$$

Integrating the previous equation in respect to $r^{\prime}$ over $R$ domain, and with respect to $\tau$ from 0 to $t^{*}=t+\varepsilon$, being $\varepsilon$ a small positive number, using Green's theorem and rearranging in order to $T(r, t)$, eqn (23) is obtained. This equation expresses the temperature field in a solid with prescribed non-homogeneous boundary conditions. The GF in the first term on the RHS should be evaluated for the time $\tau=0$. The fourth term on the RHS accounts for the effect of Dirichlet boundary conditions type. Using a classical method for solving the homogeneous problem, it is possible to determine $G\left(r, t \mid r^{\prime}, 0\right)$. Ozisik [10] stated that $G\left(r, t \mid r^{\prime}, \tau\right)$ could be obtained by replacing $t$ by $t-\tau$ in the homogeneous problem solution. 


$$
\begin{aligned}
& T(r, t)=\int_{R} G\left(r, t \mid r^{\prime}, 0\right) F\left(r^{\prime}\right) d v^{\prime}+\frac{\alpha}{k} \int_{\tau=0}^{t^{*}} \int_{R} g\left(r^{\prime}, \tau\right) G\left(r, t \mid r^{\prime}, \tau\right) d v^{\prime} d \tau \\
& +\alpha \int_{\tau=0}^{t^{*}} \sum_{i=1}^{S} \int\left[\frac{f_{i}\left(r_{i}^{\prime}, \tau\right)}{k_{i}} G\left(r, t \mid r_{i}^{\prime}, \tau\right)\right] d s_{i}^{\prime} d \tau \\
& -\left.\alpha \int_{\tau=0}^{t_{i}^{*}} \sum_{j=1}^{S} \int_{s_{j}} f_{j}\left(r_{j}^{\prime}, \tau\right) \frac{\partial G}{\partial n_{j}}\right|_{r^{\prime}=r_{j}^{\prime}} d s_{j}^{\prime} d \tau \\
& +\alpha \sum_{i=1}^{S} \int_{s_{i}} \frac{Q_{S i}}{k_{i}} G\left(r, t \mid r_{i}^{\prime}, 0\right) F\left(r_{i}^{\prime}\right) d s_{i}^{\prime}
\end{aligned}
$$

The solution of the homogeneous problem considering the homogeneous problem defined by eqns (8-11), taking into account that $F(x)=\theta_{0}$, is

$$
T(x, t)=\int_{0}^{d_{i}} \sum_{m=1}^{\infty} w(x) \frac{X_{m}\left(\beta_{m}, x\right) X_{m}\left(\beta_{m}, x^{\prime}\right)}{N\left(\beta_{m}\right)} e^{-\frac{\beta_{m}^{2} \alpha_{i}}{d_{i}^{2} t} F\left(x^{\prime}\right) d x^{\prime}}
$$

where the eigencondition is equal to $X_{m}\left(\beta_{m}, x\right)=\sin \left(\beta_{m} x / d_{i}\right)$ and the eigenvalue $\beta$ is given by the transcendental equation already presented. $w(x)$ represents the weight function equal to $w(x)=1+\left(Q_{i} / Q_{s}\right) L \delta(L-x)$ and $N\left(\beta_{m}\right)=\int_{0}^{d_{i}} w(x) X_{n}\left(\beta_{n}, x\right)^{2} d x$, Beck [9].

After substitution for the fire gas temperature in eqn (23), steel temperature will be determined according to eqn (25).

$$
T_{S t}(t)=T_{0}+\sum_{m=1}^{\infty} \sum_{j=0}^{3}\left\{\frac{1}{N\left(\beta_{m}\right)} \frac{d_{i} \sin \left(\beta_{m}\right)}{\beta_{m}\left(1-\phi_{j} d_{i}^{2} /\left(\alpha_{i} \beta_{m}{ }^{2}\right)\right)} \theta_{j}\left(e^{-\phi_{j} t}-e^{-\frac{\beta_{m}{ }^{2} \alpha_{i}}{d_{i}^{2} t}}\right)\right\}
$$

\section{Analytical and numerical comparison with experimental results}

Steel temperatures, from experimental results, described in the section 2, are compared with the analytical and numerical results. Both consider the effective thermal conductivity represented in figure 1. Since eqn (14) and eqn (25) were determined considering constant thermal properties, an incremental time stepping procedure based on a forward finite difference is applied. The remaining insulation thermal properties were considered temperature independent and equal to $\rho_{i}=1360\left[\mathrm{~kg} / \mathrm{m}^{3}\right]$ and $c_{i}=1000[\mathrm{~J} / \mathrm{kg} / \mathrm{K}]$. The steel thermal properties considered are $\rho_{S}=7850\left[\mathrm{~kg} / \mathrm{m}^{3}\right], c_{S}=600[\mathrm{~J} / \mathrm{kg} / \mathrm{K}]$ and $\lambda=45$ $[\mathrm{W} / \mathrm{m} / \mathrm{K}]$. 
Numerical results were evaluated from a 2D heat transfer model based in the finite element method performed using the PDEtool box from Matlab software. All the analyses used time increment equal to 30 [s], as recommended for numerical stability.

Figures (3-5) present the analytical and numerical steel temperature development compared with the experimental results. The solution $\left(\mathrm{T}_{\mathrm{S}}-\mathrm{DT}\right)$ and $\left(\mathrm{T}_{\mathrm{S}}-\mathrm{GF}\right)$ represents steel temperature determined by the Duhamel's theorem and Green's function methods, respectively, considering always six terms.

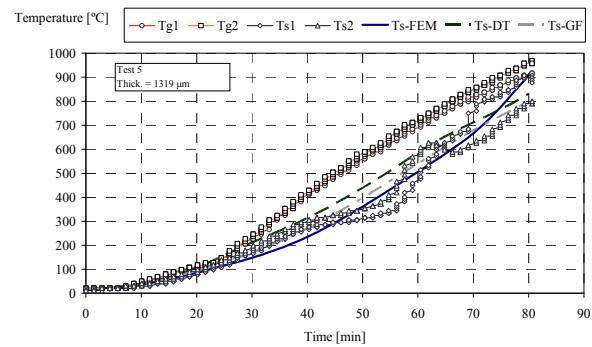

Figure 3: $\quad$ Steel temperature development from test 5.

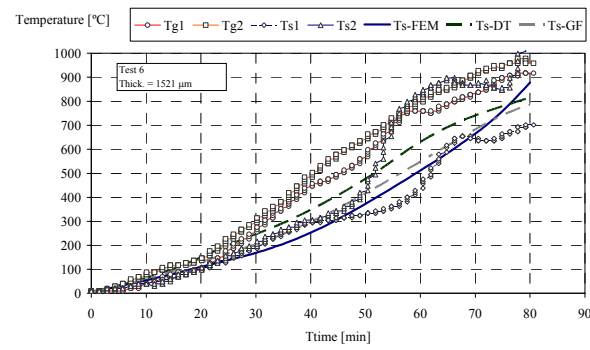

Figure 4: $\quad$ Steel temperature development from test 6.

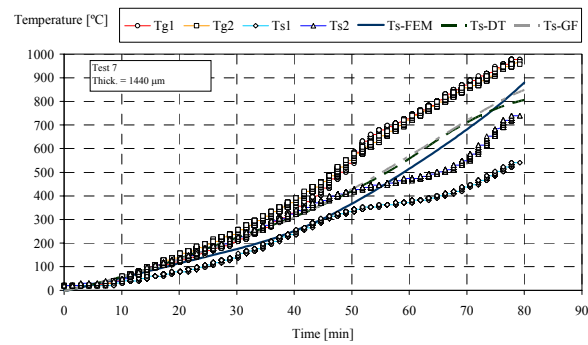

Figure 5: $\quad$ Steel temperature development from test 7.

At the initial stage all the results agree well, but for the final fire stage the analytical and the numerical results, slightly diverge.

Figures (6-8) represent steel temperature evolution for three different insulation thickness 1000, 1500 and 2000 [ $\mu \mathrm{m}]$ applied over three steel beams, HEA 300, IPE330 and a IPE160 with a section factor equal to 153, 200, and 310 $\left[\mathrm{m}^{-1}\right]$, respectively. 
For the insulation case of $1000[\mu \mathrm{m}]$, fire gas temperature and thermal properties were considered equal to the conditions of test 5 . All the other cases use both parameters equal to the conditions of test 6 .

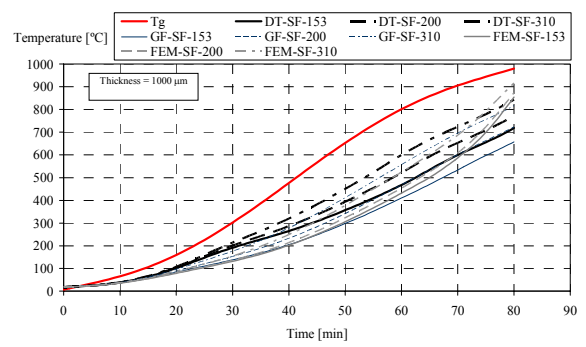

Figure 6: $\quad$ Steel temperature development for $d_{i}=1000[\mu \mathrm{m}]$.

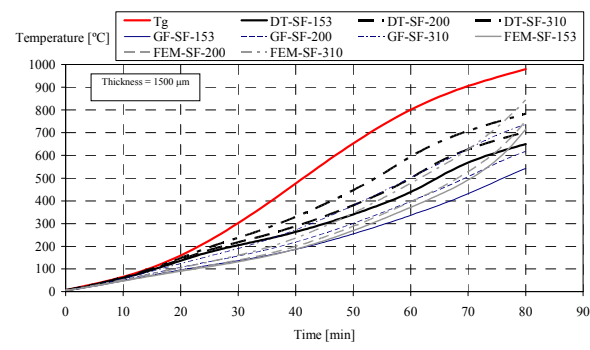

Figure 7: $\quad$ Steel temperature development for $d_{i}=1500[\mu \mathrm{m}]$.

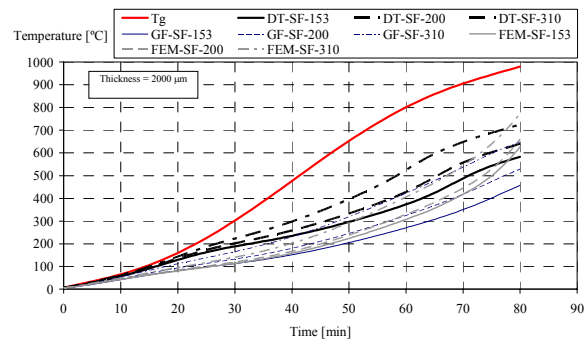

Figure 8: $\quad$ Steel temperature development for $d_{i}=2000[\mu \mathrm{m}]$.

The results, in figures $6-8$, show that for the same protection thickness, steel temperature increases with the steel section factor as expected. The results based in the Duhamel's theorem are always greater than those obtained from the Green's function. The difference between both analytical methods decrease as far as $\mu$ is reduced. This may occur by incrementing the section factor or reducing the insulation thickness.

All numerical results obtained from thermal finite element analysis are close to Green's functions results. At the final stage of simulations the numerical results and the analytical results present a distinct behaviour. 


\section{Conclusions}

Two different analytical solutions for steel temperature evolution were presented, using Duhamel's theorem and Green's function method. Both methods agree well when using six or more terms.

Non-linear numerical finite element simulations were performed using PDEtoolbox from Matlab.

Experimental results were compared with analytical and numerical predictions. Numerical results predict smaller steel temperature, in the main stage of fire, compared with analytical results.

For reduced values of the ratio of heat capacity between the insulation and steel, the results obtained from both analytical methods are close.

\section{References}

[1] Longton, P.J.; Houyoux, C.; Zhao, B.; Chico, B.; "Development of alternative technologies for off-site applied intumescent coatings", Technical Steel Research, ISBN 92-894-9165-5, 2005.

[2] Mesquita, L.M.R.; Piloto, P.A.G.; Vaz, M.A.P; Vila Real, P.M.M.; "Fire resistance tests in steel beams protected with intumescent coatings" (in Portuguese); 6th National Congress for Experimental Mechanics; vol. 12, pp 129-137, ISSN 122 922, 2006.

[3] CEN; EN 1993-1-2; "Eurocode 3, Design of Steel Structures - Part 1-2: General rules, Structural fire design"; April 2005.

[4] Wickström, U.; Temperature analysis of heavily-insulated steel structures exposed to fire; Fire of Safety Journal, 9, pp281-285, 1985.

[5] Tan, Kang Hai; Wang, Zhihua; Au, Siu Kui; "Heat transfer analysis for steelwork insulated by intumescent paint exposed to standard fire conditions"; 3rd International workshop "Structures in fire"; Ottawa Canada; May 2004.

[6] Wang, Zhi-Hua; Au, Siu Kui; Tan, Kang Hai; “ Heat Transfer analysis using a Green's function approach for uniformly insulated steel members subjected to fire"; Engineering Structures, 27, pp 1551-1562; Elsevier, 2005.

[7] Carslaw, H.S., Jaeger, J.C., "Conduction of heat in solids", Oxford University Press, Second Edition, 1959.

[8] Wang, Z.-H., Tan, K.H., "Sensitivity study of time delay coefficient of heat transfer formulations for insulated steel members exposed to fire", Fire Safety Journal, 41, 31-38, 2006.

[9] Beck, J.V., Green's Function solution for transient heat conduction problems", International Journal of Heat and Mass Transfer, vol. 27, 1235-1244, 1984.

[10] Ozisik, M.N., "Heat Conduction", John Wiley \& Sons, Inc, Second edition, 1993. 